\title{
Low-Dose Fluoroscopy Protocol for Diagnostic Cerebral Angiography
}

\author{
Yunsun Song, $\mathrm{MD}^{1}$, Seongsik Han, BS ${ }^{2}$, Byung Jun Kim, $\mathrm{PhD}^{3}$, Seong Heum Oh, BS ${ }^{2}$, Jin Su Kim, $\mathrm{BS}^{2}$, \\ Tae Il Kim, $\mathrm{BS}^{2}$, Deok Hee Lee, $\mathrm{MD}, \mathrm{PhD}^{1}$ \\ ${ }^{1}$ Department of Radiology, Research Institute of Radiology, Asan Medical Center, University of Ulsan College of Medicine, Seoul, Korea \\ ${ }^{2}$ Department of Radiologic Technology, Asan Medical Center, University of Ulsan College of Medicine, Seoul, Korea \\ ${ }^{3}$ Advanced Therapies, Siemens Healthineers Ltd., Seoul, Korea
}

Purpose: We applied a low-dose fluoroscopic protocol in routine diagnostic cerebral angiography and evaluated the feasibility of the protocol.

Materials and Methods: We retrospectively reviewed a total of 60 patients who underwent diagnostic cerebral angiography for various neurovascular diseases from September to November 2019. Routine protocols were used for patients in the first phase and low-dose protocols in the second phase. We compared radiation dose, fluoroscopy time, and complications between groups.

Results: Age, diseases, and operators were not significantly different between the two groups. The mean fluoroscopy dose significantly decreased by 52\% in the low-dose group (3.09 vs. 6.38 $\mathrm{Gy} \cdot \mathrm{cm}^{2}$ ); however, the total dose was not significantly different between the two groups (34.07 vs. $33.70 \mathrm{~Gy} \cdot \mathrm{cm}^{2}$ ). The total fluoroscopic time was slightly longer in the low-dose group, but the difference was not statistically significant (12.2. vs. 12.5 minutes). In all patients, angiography was successfully performed without complications.

Conclusion: The low-dose fluoroscopy protocol is feasible to apply for diagnostic cerebral angiography in that this protocol could significantly reduce the fluoroscopic dose.

Key Words: Cerebral angiography; Radiation; Fluoroscopy

\section{INTRODUCTION}

Despite the development of non-invasive modalities such as computed tomography and magnetic resonance imaging, diagnostic cerebral angiography remains essential for the diagnosis of many neurovascular diseases. We have been continuously applying efforts to lower the radiation dose in our hospital's neuroangiography suite. ${ }^{1,2}$ According to a previous multi-center study, our level of radiation dose during cerebral angiography and embolization procedures is one of the lowest among the hospitals in Korea. ${ }^{3}$ However, we believe that there is still scope for further dose reduction, which is attributed to fluoroscopy.

Although several studies of low-dose protocols for digital subtraction angiography (DSA) and three-dimensional rotational angiography (3D-RA) in cerebral angiography have been conducted, there have been few studies of fluoroscopic protocols due to their relatively

\section{Correspondence to:} Deok Hee Lee, MD, PhD

Department of Radiology, Research Institute of Radiology, Asan Medical Center, University of Ulsan College of Medicine, 88 Olympic-ro 43-gil, Songpa-gu, Seoul 05505, Korea

Tel: +82-2-3010-5944

Fax: +82-2-476-0090

E-mail:dhlee@amc.seoul.kr

Received: April 3, 2020

Revised: May 6, 2020

Accepted: May 6, 2020
Copyright $\odot 2020$ Korean Society of Interventional Neuroradiology This is an Open Access article distributed under the terms of the Creative Commons Attribution Non-Commercial License (http://creativecommons.org/licenses/by-nc/4.0) which permits unrestricted non-commercial use, distribution, and reproduction in any medium, provided the original work is properly cited.

pISSN 2093-9043 eISSN 2233-6273 
low proportion in total radiation dose and the difficulty in evaluating image quality. ${ }^{4-11}$ In the case of cardiac intervention, where fluoroscopy plays a main role, various protocols have been investigated to achieve a reduction in fluoroscopic dose, including the effects of detector entrance dose (DED), frame rate, copper filter, peak kilovoltage (kVp), pulse width, focal spot size, and fluoroscopy time limitation. ${ }^{12-15}$ Most of these studies have concluded that radiation dose could be reduced without complications or procedural delays.

In our angiography suite, therefore, several low-dose protocols for fluoroscopy have been applied to achieve a reduction in radiation exposure, including a limitation of DED or kVp and adding a copper filter in routine diagnostic cerebral angiography. Among the factors, we decided to lower the DED for this study. The reason was that the evaluation of fluoroscopic image quality was not clear enough to discriminate between the merits and demerits of several different factors. Also, it was difficult to optimize them.

The aim was to prove that applying a low-dose fluoroscopic protocol leads to a significant reduction of fluoroscopic dose and total radiation dose during routine diagnostic cerebral angiography without procedural prolongation or compromising patients' safety.

\section{MATERIALS AND METHODS}

\section{Patients}

We applied low-dose fluoroscopic protocols for diagnostic cerebral angiography in one of our angiography rooms since October 2019 as part of quality control activity for radiation dose and image quality. In this retrospective study, we included 30 consecutive patients who underwent diagnostic cerebral angiography with the low-dose protocols for various cerebrovascular diseases between October and November 2019. Additionally, we included 30 consecutive patients as a control group who were examined using the routine protocol in September 2019. We excluded patients who underwent spinal angiography, pediatric patients (aged $\leq 18$ years), patients who had a high-density prosthesis (gamma knife frame or embolic material) in the head, and patients for whom the radiation dose reports were unavailable. The Asan Medical Center Institutional Review Board approved this study (approval number: 2020-0418) and waived the requirement to obtain written, informed consent from patients.

\section{Imaging equipment and protocols}

All angiography data were obtained using a biplane angiography machine (Artis Zee; Siemens, Forchheim, Germany). The DED for DSA was $1.82 \mu \mathrm{Gy} /$ frame. We used variable DSA frames per second (f/s) containing 4 phases (phase 1: $2 \mathrm{f} / \mathrm{s}$ for $1 \mathrm{~s}$; phase 2: $2 \mathrm{f} / \mathrm{s}$ for 1 second, phase 3: $1 \mathrm{f} / \mathrm{s}$ for 2 seconds, phase $4: 0.5 \mathrm{f} / \mathrm{s}$ for the remainder of the acquisition, up to 40 seconds). The DED for 3D-RA was $0.24 \mu \mathrm{Gy} /$ frame. Copper filters were automatically applied in the range of 0.1 to 0.3 $\mathrm{mm}$ for fluoroscopy and DSA and not applied for 3D-RA. The focal spot size was $0.3 \mathrm{~mm}$ (micro) for DSA and fluoroscopy and $0.4 \mathrm{~mm}$ (small) for 3D-RA. The C-arm angles, table position, collimation, magnification, and source-image distance, which may affect the radiation dose, were determined at the discretion of the operator under the basic rules of the "as low as reasonably achievable" principle. The frame rate for fluoroscopy was set to a standard of $7.5 \mathrm{f} / \mathrm{s}$. The $\mathrm{kVp}$, milliampere $(\mathrm{mA})$, pulse width, and copper filter were automatically determined by the angiographic system in the fixed routine protocol.

The low-dose fluoroscopic protocols were generated by stepping down from a routine DED of 23 nGy per pulse (nGy/p) to $18,15,12,10$, and $8 \mathrm{nGy} / \mathrm{p}$ for fluoroscopy and $36 \mathrm{nGy} / \mathrm{p}$ to $28,23,18,15$, and $12 \mathrm{nGy} / \mathrm{p}$ for roadmap imaging. The examination was initiated at a minimum of $8 \mathrm{nGy} / \mathrm{p}$ (roadmap $12 \mathrm{nGy} / \mathrm{p}$ ), and the operator was permitted to choose a higher DED when any difficulties were encountered in the procedure (Fig. 1).

All examinations were performed by 3 operators with 4 years (A), 1 year (B), and 2 months (C) of neurointervention experience, respectively. Of the 30 patients in the routine-dose group, 10, 9, and 11 patients underwent examinations performed by operators $A, B$, and $C$, respectively. Among the 30 patients in the low-dose group, 15, 4, and 11 patients underwent examinations performed by operators $A, B$, and $C$, respectively. There was no statistical difference in this distribution.

\section{Definitions}

Radiation dose reports were reviewed for radiation dose metrics: reference air kerma (Ka,r in mGy) and kerma-area product (PKA in $\mathrm{Gy} \cdot \mathrm{cm}^{2}$ ), fluoroscopic time, and the number of exposures. The total radiation dose of cerebral angiography consists of 3 items presented in the dose report: fluoroscopy, DSA, and 3D-RA. The fluoroscopic dose is the sum of the radiation dose during the use of fluoroscopy. DSA and 

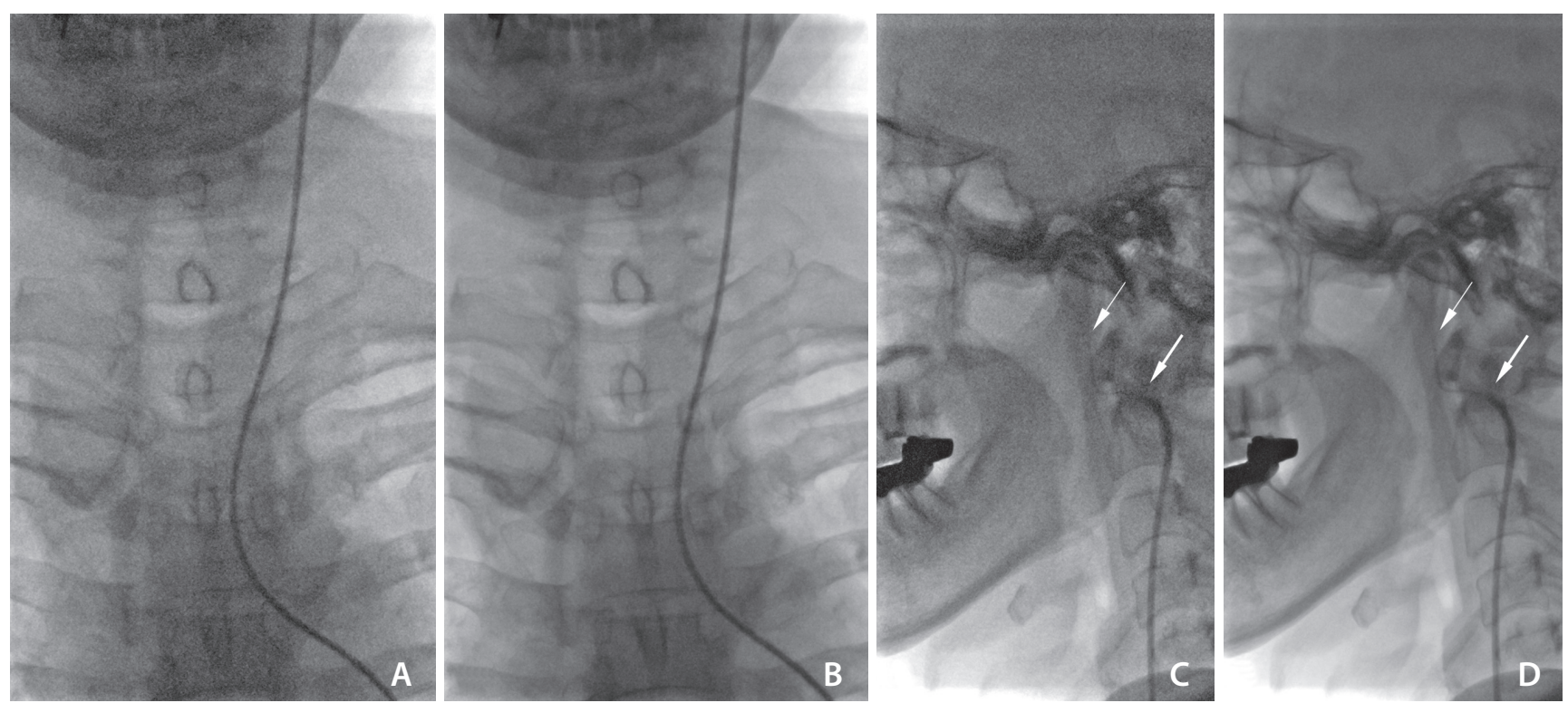

Fig. 1. Visual comparison between low-dose and routine-dose protocols. Fluoroscopic still images in biplane views are shown in the same patient during left internal cerebral artery selection. A 5-French catheter and a 0.032-inch guidewire were used. The tips of the catheter (thick arrows) and guidewire (thin arrows) are visualized in both protocols. (A, C) Low-dose protocol (8 nGy/pulse); (B, D) Routine dose protocol (23 nGy/pulse).

3D-RA dose are the radiation doses of 2-dimensional (2D) and 3D rotational acquisitions, respectively. We analyzed each item and the total between the 2 groups. Fluoroscopic time is the total time of fluoroscopy, also known as the "pedal time" or the time during which the operator presses the fluoroscopy pedal. The number of exposures is the combined number of $2 \mathrm{D}$ and $3 \mathrm{D}$ acquisitions, and it counts as 2 for biplane 2D DSA run, and 2 for 3D DSA mode (2 rotations without and with a contrast agent, sequentially).

\section{Cerebral angiography}

We routinely used the femoral artery approach using a 4 French (F) sheath, while we used a 5-F long sheath when the iliac artery was markedly tortuous. lliac arteriography was not routinely performed. The routine diagnostic catheters were 4-F Davis or Headhunter catheters initially; these were changed to Simmons 2 catheters in cases of a type 3 aortic arch. Using these catheters, we routinely obtained roadmap images of the targeted neck vessels before guidewire advancement (Supplementary Videos 1-7).

Routine cerebral angiography, which consisted of bilateral internal carotid artery (ICA) and vertebral artery angiograms, and 3D-RA for the target lesion were performed in all patients. External carotid artery angiograms were selectively performed for arteriovenous shunt lesions, severe intracranial stenosis, and pre- or post-operative evaluation of bypass sur- gery. In cases of severe bulb stenosis or tortuous neck vessel anatomy, common carotid artery angiograms were used instead of ICA angiograms.

Electronic medical records were reviewed for patients' baseline characteristics such as age and underlying disease, number of vessels catheterized, and procedure-related complications, including symptomatic thromboembolism, arterial dissection, and puncture site problems.

\section{Statistical considerations}

Statistical calculations were computed using STATA version 13.0 (StataCorp LP, College Station, TX, USA). Continuous variables were expressed as means \pm standard deviations. Categorical variables were expressed as frequencies with percentages. The statistical significance between dose parameters in the low-dose and routine-dose groups was determined using the Student's t-test or Wilcoxon signedrank test (if the assumption of normality was violated on the Shapiro-Wilk normality test) for continuous measures. Analysis of the underlying disease type and operator distribution in each group was performed using Fisher's exact test. Pearson's product-moment correlation was determined to assess the relationship between the total fluoroscopic time and age of the patients. A P-value $<0.05$ was considered to be statistically significant. 


\section{RESULTS}

The mean age of the patients was 56 years, without a significant difference between the low-dose and routine-dose groups. Underlying vascular diseases were divided into aneurysms, stenosis, shunt lesions, tumors, and normal; there was no significant difference between the 2 groups (Table 1).

The mean fluoroscopy dose significantly decreased by $52 \%$ in the low-dose group compared to that in the routine-dose group (3.09 vs. $6.38 \mathrm{~Gy} \cdot \mathrm{cm}^{2}, \mathrm{P}=0.005$ ). This dose reduction was more pronounced in the lateral tube than in the frontal tube (Fig. 2). On the other hand, the average DSA and 3D-RA doses were $8.9 \%$ and $31 \%$ higher in the low-dose group, respectively; thus, the total dose was slightly higher in the lowdose group, but there was no significant difference (34.07 vs. 33.70 Gy. $\mathrm{cm}^{2}, \mathrm{P}=0.88$ ) (Fig. 3).

The mean fluoroscopic times of the frontal and lateral tubes for each group are shown in Fig. 4. In both groups, the frontal tube time was twice as long as the lateral tube time. In the low-dose group, the mean frontal tube time was slightly longer, whereas the lateral tube time was shorter. Overall, the total fluoroscopic time was slightly longer in the low-dose group (12.2. vs. 12.5 minutes, $P=0.65$ ).

There was a moderate positive correlation between the total fluoroscopic time and age ( $r=0.401, P<0.0015)$ (Fig. 5). In the subgroup analysis of patients aged older than 60 years, the mean fluoroscopic time (12.4 vs. 7.9 minutes) and dose (5.73 vs. $3.87 \mathrm{~Gy} \cdot \mathrm{cm}^{2}$ ) increased by 50\% compared to those in patients aged younger than 60 years. The proportion of the fluoroscopy increased to up to $23 \%$ of the total dose in the routine-dose group and $11 \%$ in the low-dose group, which were $14 \%$ and $7 \%$ in young patients, respectively.

As a result, cerebral angiography was successfully performed in all patients included in the study. Of the 2 patients in the low-dose group, the DED increased from $8 \mathrm{nGy} / \mathrm{p}$ to $12 \mathrm{nGy} / \mathrm{p}$ at the discretion of the operator during catheterization in the aortic arch. There were no complications associated with the procedure in all patients.

\section{Table 1. Patient demographics and procedural data}

\begin{tabular}{|c|c|c|c|}
\hline Protocol & Low dose $(n=30)$ & Routine $(n=30)$ & P-value \\
\hline Age (y) & $57.4 \pm 13.7$ & $54.6 \pm 15.8$ & $0.45^{*}$ \\
\hline Underlying diseases & & & $0.28^{\ddagger}$ \\
\hline Aneurysm & $15(50.0)$ & $17(56.7)$ & \\
\hline Stenosis & $9(30.0)$ & $5(16.7)$ & \\
\hline Shunt lesion & $0(0.0)$ & $3(10)$ & \\
\hline Tumor & $5(16.7)$ & $5(16.7)$ & \\
\hline Normal & $1(3.3)$ & $0(0.0)$ & \\
\hline Number of vessels & $4.6 \pm 1.9$ & $4.7 \pm 1.4$ & $0.75^{*}$ \\
\hline Number of exposures & $10.4 \pm 3.1$ & $10.1 \pm 2.3$ & $0.71^{*}$ \\
\hline Total fluoroscopic time (min) & $10.2 \pm 5.1$ & $9.8 \pm 5.1$ & $0.65^{\dagger}$ \\
\hline \multicolumn{4}{|l|}{ Radiation dose } \\
\hline Fluoroscopy (DAP, $\mu$ Gym²) & $309 \pm 183.7$ & $638.4 \pm 390.5$ & $0.005^{+}$ \\
\hline Fluoroscopy (AK, mGy) & $26 \pm 14.6$ & $55.5 \pm 33.7$ & $0.005^{+}$ \\
\hline DSA (DAP, $\left.\mu G_{y m}^{2}\right)$ & $2,355.4 \pm 910.8$ & $2,163.2 \pm 779.1$ & $0.33^{+}$ \\
\hline DSA (AK, mGy) & $149.1 \pm 57.2$ & $139.6 \pm 47.1$ & $0.49^{*}$ \\
\hline 3D-RA (DAP, $\mu \mathrm{Gym}^{2}$ ) & $742.6 \pm 521.2$ & $568.1 \pm 326.2$ & $0.12^{*}$ \\
\hline 3D-RA (AK, mGy) & $80.6 \pm 57.1$ & $61.8 \pm 35.9$ & $0.13^{*}$ \\
\hline Total (DAP, $\mu G_{y m}^{2}$ ) & $3,407 \pm 888.6$ & $3,369.7 \pm 1,039.8$ & $0.88^{*}$ \\
\hline Total (AK, mGy) & $255.6 \pm 70.1$ & $256.9 \pm 67.5$ & $0.94^{*}$ \\
\hline
\end{tabular}

Data are presented as mean \pm standard deviation or number (\%).

DAP, dose area product; AK, air kerma; DSA, digital subtraction angiography; 3D-RA, three-dimensional rotational angiography.

${ }^{*}$ t-test or ${ }^{\dagger}$ Wilcoxon rank-sum test for continuous measures. ${ }^{\ddagger}$ Fisher's exact test for categorical variables. 


\section{DISCUSSION}

In this patient study, the use of low-dose fluoroscopy protocols in cerebral angiography demonstrated a reduction in fluoroscopic dose by almost half. In addition, the examination was completed without a prolonged fluoroscopy time or complications associated with the procedure.

The application of the low-dose fluoroscopy protocol did not result in a significant total dose reduction in this study. This is mainly due to the relatively low proportion (18.9\%) of fluoroscopy in diagnostic cerebral angiography in our study.

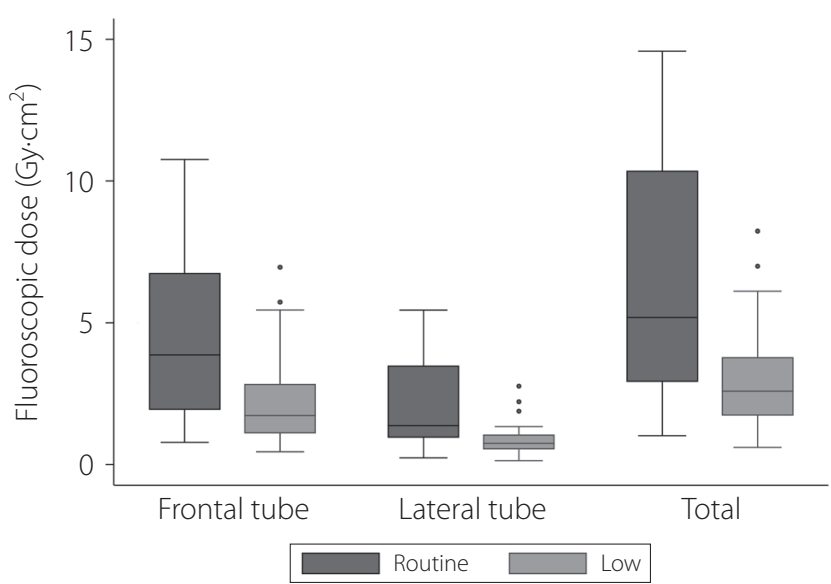

Fig. 2. Box plots of fluoroscopic dose (kerma-area product) between routine and low dose groups for each tube. The top of the box represents the 75th percentile, the bottom of the box represents the 25th percentile, and the line in the middle represents the 50th percentile. The whiskers represent the highest and lowest values that are not outliers or extreme values. Circles beyond the whiskers represent outliers and extreme values.

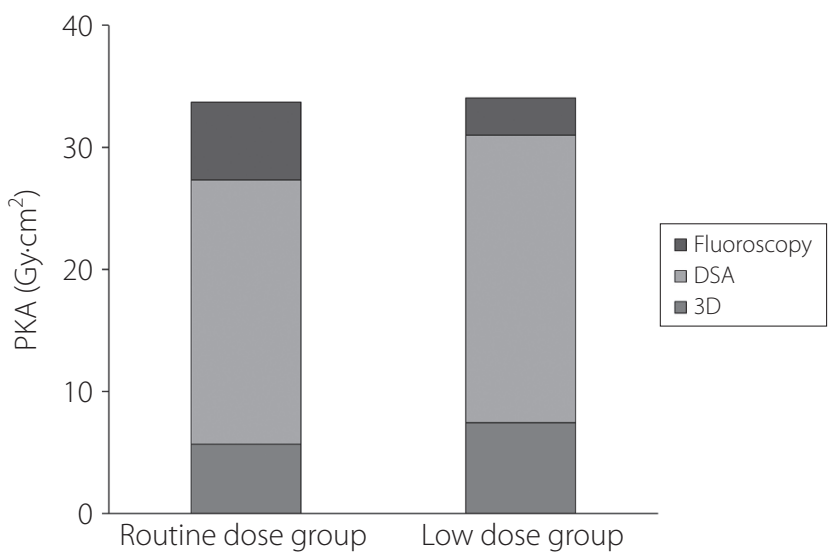

Fig. 3. Total radiation dose comparison and composition. PKA, kerma-area product; DSA, digital subtraction angiography; 3D, three-dimensional.
Another reason was that increased 3D-RA and DSA doses outweighed the reduction of fluoroscopic dose in the lowdose group. If other conditions were the same, about a 10\% total dose reduction would be expected.

Fluoroscopy time is not the only factor directly associated with fluoroscopy dose but can also be a result of low-dose protocols; for example, a longer fluoroscopy time may be the effect of poor image quality. In the low-dose group in our study, the observation of slight increases in frontal fluoroscopy time and stepping up of the DED in 2 patients during

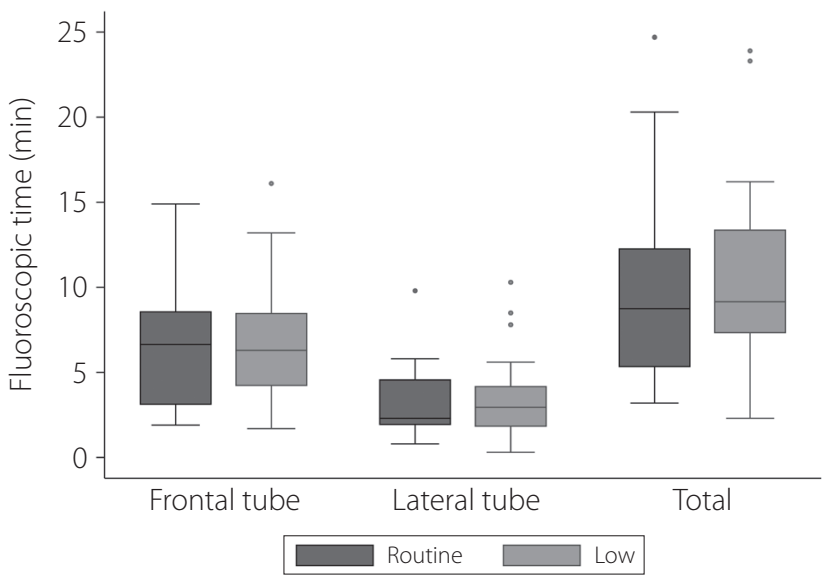

Fig. 4. Box plots of fluoroscopic time between routine and low dose groups for each tube. The top of the box represents the 75th percentile, the bottom of the box represents the 25th percentile, and the line in the middle represents the 50th percentile. The whiskers represent the highest and lowest values that are not outliers or extreme values. Circles beyond the whiskers represent outliers and extreme values.

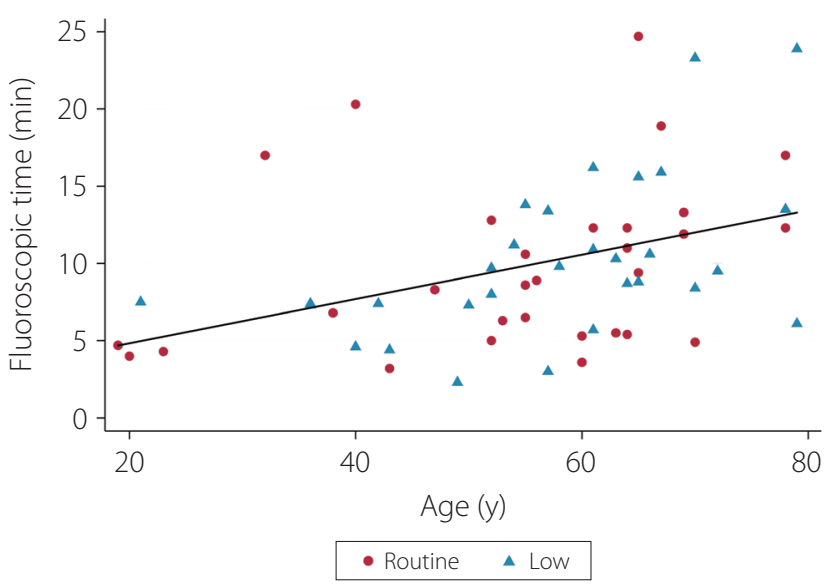

Fig. 5. Scatter plot of the fluoroscopic time and age of the patients showing the best-fit simple regression line. The red circle indicates the routine group $(n=30)$, and the blue triangle indicates the low dose group $(n=30)$. The solid line is the linear regression line for the entire group. 
aortic arch vessel selection may raise concerns about image quality, especially in the chest. However, there was no significant increase in fluoroscopy time due to the use of low-dose protocols in this study.

The positive correlation between the patient's age and fluoroscopy time was likely due to a longer catheter manipulation time as the neck vessels become tortuous with age. A similar trend was observed in a study that applied a dose reduction strategy during cerebral angiography wherein the total fluoroscopic times were 5.8 minutes for patients aged younger than 50 years and 9.5 minutes for patients aged older than 50 years. ${ }^{6}$ Therefore, the effect of the low-dose protocol is expected to be relatively high in elderly patients.

The strength of this study is that no previous study has focused on fluoroscopy in the neurovascular field. Although the low-dose fluoroscopic protocol in diagnostic cerebral angiography did not result in a great clinical significance, it may be meaningful if applied to complex neurovascular procedures such as embolization of brain arteriovenous malformations (AVM) or dural arteriovenous fistulas, which are associated with a high proportion of performing fluoroscopy. Of course, the image quality required for the procedure should be better than that of angiography, but according to a previous study using a low-dose fluoroscopic protocol for peripheral AVM, the image quality was satisfactory and the dose decreased by more than $80 \%{ }^{16}$ As for the neurovascular procedure, further research will be needed on a low-dose protocol with a good balance of image quality and dose.

There are several limitations to this study. First, the image quality of the low-dose protocol was not evaluated. The reason is that, unlike DSA, which is generally assessed by still images, fluoroscopy should be evaluated by viewing the video, which is challenging to be done reliably and objectively. Instead, the fluoroscopic time and the incidence of complications determined the adequacy of the image quality indirectly. Second, due to difficulty in optimizing parameters that interact with each other, such as kVp and a copper filter, they could not be adjusted together. Further studies with phantoms or collaborations with the vendor may be able to accomplish this. Lastly, there was an imbalance in the operator distribution, 3D-RA dose, and DSA dose between the groups due to the retrospective design. A prospective study with well-matched groups would more clearly elucidate the effect of low-dose fluoroscopy protocols on the total radiation dose.

\section{CONCLUSION}

A low-dose fluoroscopy protocol was effective and safe in reducing fluoroscopic doses in diagnostic cerebral angiography. Although the low-dose fluoroscopy protocol alone did not result in a significant reduction in total dose, it may be helpful for further dose reduction when used with low-dose DSA or 3D protocols. It may also be effective in the elderly or in patients with tortuous anatomy who require longer fluoroscopy time.

\section{SUPPLEMENTARY MATERIALS}

Supplementary materials related to this article can be found online at https://doi.org/10.5469/neuroint.2020.00129.

\section{Fund}

This study was supported by the Korean Society of Interventional Neuroradiology (KSIN) research grant 2019.

\section{Ethics Statement}

This study was approved by the Institutional Review Board of Asan Medical Center.

\section{Conflicts of Interest}

The authors have no conflicts to disclose.

\section{Author Contribution}

Concept and design: YS, SH, BJK, JSK, TIK, and DHL. Analysis and interpretation: YS, SH, BJK, and SHO. Data collection: YS, $\mathrm{SH}$, and SHO. Writing the article: YS. Critical revision of the article: YS and DHL. Final approval of the article: YS and DHL. Statistical analysis: YS. Obtained funding: YS. Overall responsibility: DHL.

\section{ORCID}

Yunsun Song: https://orcid.org/0000-0003-4738-0533

Seongsik Han: https://orcid.org/0000-0002-9873-674X

Byung Jun Kim: https://orcid.org/0000-0003-1720-1012

Seong Heum Oh: https://orcid.org/0000-0002-1128-5895

Jin Su Kim: https://orcid.org/0000-0001-7797-1401

Tae II Kim: https://orcid.org/0000-0001-7839-196X

Deok Hee Lee: https://orcid.org/0000-0003-0355-0449 


\section{REFERENCES}

1. Song Y, Kim Y, Han S, Kim TI, Choi JH, Maeng JY, et al. Estimated radiation dose according to the craniocaudal angle in cerebral digital subtraction angiography: patient and phantom study. J Neuroradio/ 2019:46:345-350

2. Maeng JY, Song Y, Sung YS, Kim TI, Lee DH, Kim TH. Feasibility of ultra-low radiation dose digital subtraction angiography: preliminary study in a simplified cerebral angiography phantom. Interv Neuroradiol 2019;25:589-595

3. Ihn YK, Kim BS, Byun JS, Suh SH, Won YD, Lee DH, et al. Patient radiation exposure during diagnostic and therapeutic procedures for intracranial aneurysms: a multicenter study. Neurointervention 2016;11:78-85

4. Riabroi K, Khanungwanitkul K, Wattanapongpitak P, Krisanachinda A, Hongsakul K. Patient radiation dose in neurointerventional radiologic procedure: a tertiary care experience. Neurointervention 2018;13:110-116

5. Yi HJ, Sung JH, Lee DH, Kim SW, Lee SW. Analysis of radiation doses and dose reduction strategies during cerebral digital subtraction angiography. World Neurosurg 2017;100:216-223

6. Schneider T, Wyse E, Pearl MS. Analysis of radiation doses incurred during diagnostic cerebral angiography after the implementation of dose reduction strategies. J Neurointerv Surg 2017;9:384-388

7. Kim DJ, Park MK, Jung DE, Kang JH, Kim BM. Radiation dose reduction without compromise to image quality by alterations of filtration and focal spot size in cerebral angiography. Korean J Radiol 2017;18:722-728

8. Honarmand AR, Shaibani A, Pashaee T, Syed FH, Hurley MC, Sammet $C L$, et al. Subjective and objective evaluation of image quality in biplane cerebral digital subtraction angiography following significant acquisition dose reduction in a clinical set- ting. J Neurointerv Surg 2017;9:297-301

9. Kahn EN, Gemmete JJ, Chaudhary N, Thompson BG, Chen K, Christodoulou EG, et al. Radiation dose reduction during neurointerventional procedures by modification of default settings on biplane angiography equipment. J Neurointerv Surg 2016:8:819-823

10. Pearl MS, Torok C, Wang J, Wyse E, Mahesh M, Gailloud P. Practical techniques for reducing radiation exposure during cerebral angiography procedures. J Neurointerv Surg 2015;7:141-145

11. Alexander MD, Oliff MC, Olorunsola OG, Brus-Ramer M, Nickoloff EL, Meyers PM. Patient radiation exposure during diagnostic and therapeutic interventional neuroradiology procedures. $J$ Neurointerv Surg 2010;2:6-10

12. Plank F, Stowasser B, Till D, Schgör W, Dichtl W, Hintringer F, et al. Reduction of fluoroscopy dose for cardiac electrophysiology procedures: a feasibility and safety study. Eur J Radiol 2019;110:105-111

13. Crowhurst J, Haqqani H, Wright D, Whitby M, Lee A, Betts J, et al. Ultra-low radiation dose during electrophysiology procedures using optimized new generation fluoroscopy technology. Pacing Clin Electrophysiol 2017;40:947-954

14. Attanasio P, Mirdamadi M, Wielandts JY, Pieske B, Blaschke F, Boldt LH, et al. Safety and efficacy of applying a low-dose radiation fluoroscopy protocol in device implantations. Europace 2017;19:1364-1368

15. Lee SY, Kim J, Lee SH, Choi JH, Kim JS, Park YH, et al. Reduction of radiation exposure during catheter ablation for paroxysmal supraventricular tachycardia: the effect of a low frame rate of digital pulsed fluoroscopy. Int J Cardiol 2014;177:573-574

16. Wildgruber M, Köhler M, Brill R, Goessmann H, Uller W, MüllerWille $R$, et al. Impact of low dose settings on radiation exposure during pediatric fluoroscopic guided interventions Eur J Radiol 2018:100:1-6 